\title{
Draft genome of the emerging pathogen, Kocuria marina, isolated from a wild urban rat
}

\author{
Shih Keng Loong1, Kim-Kee Tan ${ }^{1,2}$, Nurhafiza Zainal ${ }^{1,2}$, Wai Hong Phoon ${ }^{1,2}$, \\ Siti Nursheena Mohd Zain ${ }^{3}$, Sazaly AbuBakar ${ }^{1,2}+$ \\ ${ }^{1}$ University of Malaya, Faculty of Medicine, Tropical Infectious Diseases Research \& Education Centre, Kuala Lumpur, Malaysia \\ ${ }^{2}$ University of Malaya, Faculty of Medicine, Department of Medical Microbiology, Kuala Lumpur, Malaysia \\ ${ }^{3}$ University of Malaya, Faculty of Science, Institute of Biological Sciences, Kuala Lumpur, Malaysia
}

Kocuria marina has recently emerged as a cause for catheter-related bloodstream infections in patients with underlying health complications. One K. marina strain was recently isolated from the lung tissues of a wild urban rat (Rattus rattus diardii) caught during rodent surveillance. Here, we present the draft genome of the first $K$. marina animal isolate, K. marina TRE150902.

Key words: infectious disease - Kocuria - zoonosis - Malaysia - Rattus rattus

Kocuria marina is a Gram positive cocci, isolated initially from marine sediment (Kim et al. 2004) and shares identical morphology to Staphylococci and Micrococci (Kandi et al. 2016). Although predominantly found in the environment and, as normal flora on the skin and oropharynx of mammals, clinical cases are increasingly described (Purty et al. 2013), signifying the pathogenic potential of this bacterial species. Clinical cases reported in the literature involved the elderly and the young with underlying health complications and were mostly associated with catheter usage (Lee et al. 2009, Lai et al. 2011, Brändle et al. 2014, Horiuchi et al. 2015, Mori et al. 2017). $K$. marina was also recently isolated from the lung tissues of a wild urban rat (Loong et al. 2016), potentially adding a zoonotic dimension into the transmission cycle.

Here, we present the draft genome of $K$. marina TRE150902 isolated from rat lung tissues. Strain TRE150902 was susceptible to common antibiotics and was confirmed as K. marina by $16 \mathrm{~S}$ rDNA sequencing and other phenotypic tests (Loong et al. 2016). Strain TRE150902 was cultured overnight in brain-heart infusion broth under aerobic conditions at $37^{\circ} \mathrm{C}$ and, the resulting bacterial culture was used for transmission electron microscopy (Tan \& Suresh 2006) and genome sequencing.

Bacterial cells from late-exponential phase were fixed overnight at $4{ }^{\circ} \mathrm{C}$ with $4 \%$ (vol $/ \mathrm{vol}$ ) glutaraldehyde and $0.1 \mathrm{M}$ sodium cacodylate buffer. The bacterial cells were subsequently washed with sterile water and dehydrated using increasing concentrations of ethanol. Following that, bacterial cells were embedded overnight in epoxy resin and then sliced into ultrathin sections using a diamond knife (Diatome, USA). Sections were mount-

doi: 10.1590/0074-02760170132

Financial support: University of Malaya Research Grant (RP016C-14AFR),

High Impact Research-Ministry of Higher Education Grant (E000013-20001).

+ Corresponding author: sazaly@um.edu.my

Received 2 April 2017

Accepted 8 June 2017 ed on a mesh copper grid (Ted Pella, USA), stained with uranyl acetate and then imaged using a HT7700 Transmission Electron Microscope (Hitachi, Japan).

Whole genome sequencing of $K$. marina TRE150902 was performed as previously described (Tan et al. 2015, Loong et al. 2017) with minor modifications. Genome library preparation was performed using Ion Xpress Plus Fragment Library Kit (Thermo Fisher Scientific, USA) and genome libraries corresponded to 200bp were prepared using E-Gel SizeSelect Agarose Gel, 2\% (Thermo Fisher Scientific, USA). The sequencing template was prepared using Ion PGM Hi-Q OT2 Kit (Thermo Fisher Scientific, USA) according to manufacturer's protocol. Amplified Ion Sphere Particles were enriched using Ion PGM Enrichment beads (Thermo Fisher Scientific, USA) and genome sequencing was undertaken using the Ion Torrent PGM sequencer (Life Technologies, USA) using Ion PGM Hi-Q sequencing kit. The Ion Torrent reads were assembled de novo using SPAdes v3.1.0 (Bankevich et al. 2012) and the assembled contigs were functionally annotated with Rapid Annotation using Subsystem Technology (RAST) (Aziz et al. 2008).

Transmission electron microscopy revealed that the morphological characteristics of $K$. marina resembled Staphylococcus aureus (Touhami et al. 2004), showing observable growth and division by the formation of septum (Figure). The resulting draft genome of $K$. marina TRE150902 was 2,856,751 bp in length, comprising of 54 contigs and $\mathrm{N}_{50}$ of 98,614 . The GC content of the draft genome was approximately $68.9 \%$ and a total 2607 protein-coding genes with 50 RNAs were predicted using RAST. These results were summarised in Table.

Genome annotation in the RAST server uncovered the presence of genes coding for enzymes involved in invasion and intracellular resistance, which include quinolinate synthetase (EC 2.5.1.72), quinolinate phosphoribosyltransferase [decarboxylating] (EC 2.4.2.19), Laspartate oxidase (EC 1.4.3.16), inner membrane protein translocase component YidC, protein YidD and RNAbinding protein Jag. In addition, genes responsible for 


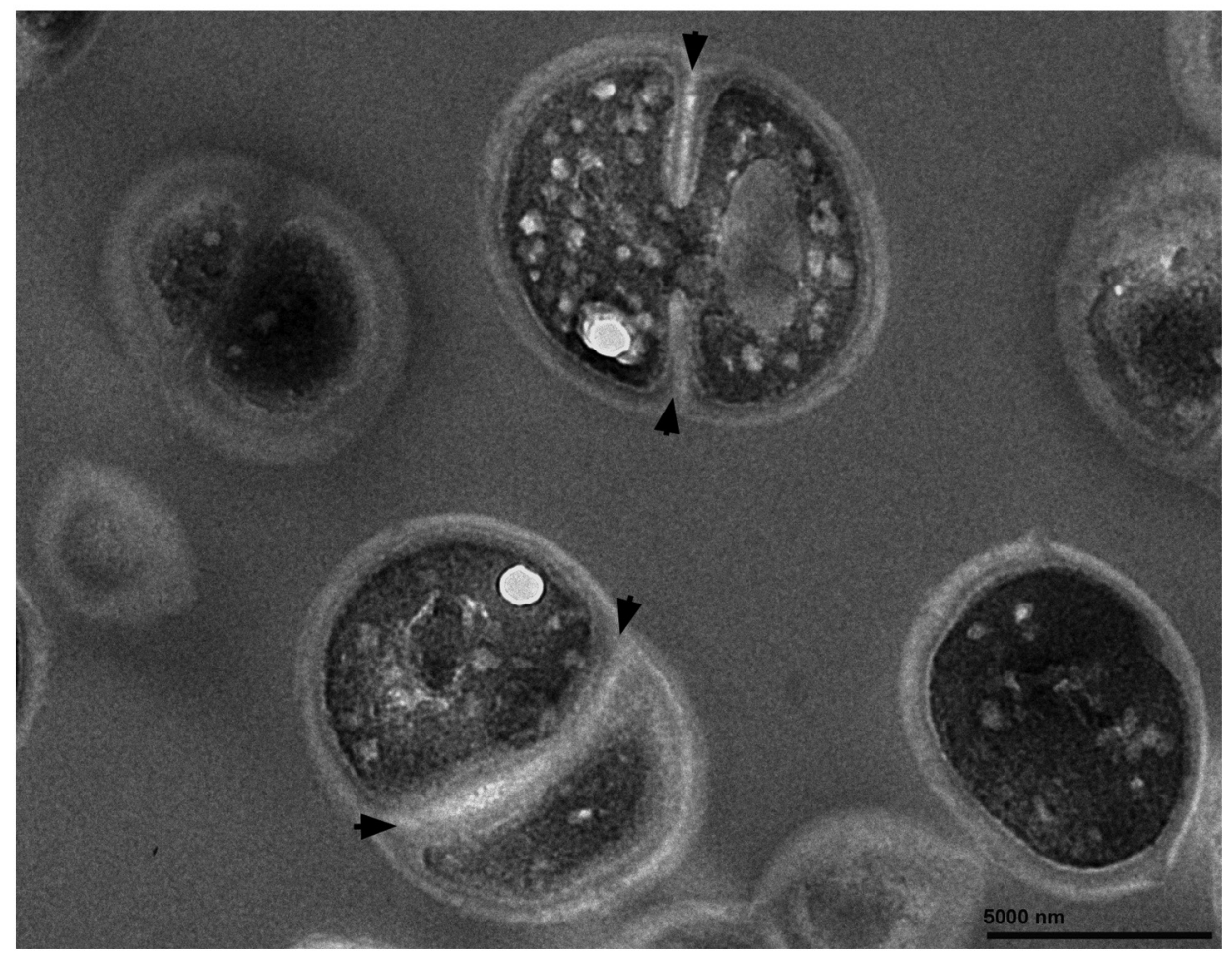

Morphological characteristics of Kocuria marina TRE150902 as shown by transmission electron microscopy at 12,000 times magnification. Arrows show the formation of septa.

TABLE

Overview of Kocuria marina TRE150902 genome assembly

\begin{tabular}{lc}
\hline Attribute & Chromosome \\
\hline Genome size (bp) & $2,856,751$ \\
GC content (\%) & 68.9 \\
Contigs & 54 \\
Open reading frames & 2697 \\
RNA & 50 \\
\hline
\end{tabular}

resistance to toxic compounds (mercuric ion reductase (EC 1.16.1.1), copper resistance protein CopC, cobaltzinc-cadmium resistance protein $\mathrm{CzcD}$ and arsenical-resistance protein ACR3) were also annotated, potentially aiding K. marina TRE150902 proliferation in various environments (Purty et al. 2013), outside the host.

The potential zoonotic transmission of K. marina which otherwise known to be transmitted only from the environment to humans would undoubtedly confound disease and risk management of the bacteria. These highlight the emerging complexity of interactions between human, animal, environmental factors and new bacterial pathogens. The draft genome of $K$. marina TRE150902 will aid genomic analyses and comparison with other clinical and environmental strains to elucidate the potential diversity between them. The draft genome sequences generated in this study are available from the European Nucleotide Archive under the study number PRJEB19997.

\section{AUTHORS' CONTRIBUTION}

SKL, KKT and NZ - Involved in the sequencing of the genome; SKL and WHP - involved in transmission electron microscopy; SKL, SNMZ and SA - involved in bacteria isolation and interpretation of the data; SKL and SA - designed the study, obtained funding and wrote the paper. All authors read and approved the final manuscript.

\section{REFERENCES}

Aziz RK, Bartels D, Best AA, Dejongh M, Disz T, Edwards RA, et al. The RAST server: rapid annotations using subsystems technology. BMC Genomics. 2008; 9: 75.

Bankevich A, Nurk S, Antipov D, Gurevich AA, Dvorkin M, Kulikov AS, et al. SPAdes: a new genome assembly algorithm and its applications to single-cell sequencing. J Comput Biol. 2012; 19(5): 455-77.

Brändle G, L'Huillier AG, Wagner N, Gervaix A, Wildhaber BE, Lacroix L. First report of Kocuria marina spontaneous peritonitis in a child. BMC Infect Dis. 2014; 14: 719.

Horiuchi A, Kubota N, Hidaka E, Shimabukuro A, Yasukochi S, Nakamura T, et al. Notable alkaline tolerance on Kocuria marina isolate from blood of a pediatric patient with continuous intravenous epoprostenol therapy. J Infect Chemother. 2015; 21(9): 680-6.

Kandi V, Palange P, Vaish R, Bhatti AB, Kale V, Kandi MR, et al. Emerging bacterial infection: identification and clinical significance of Kocuria species. Cureus. 2016; 8(8): e731.

Kim SB, Nedashkovskaya OI, Mikhailov VV, Han SK, Kim KO, Rhee MS, et al. Kocuria marina sp. nov., a novel actinobacterium 
isolated from marine sediment. Int J Syst Evol Microbiol. 2004; 54(Pt 5): 1617-20.

Lai CC, Wang JY, Lin SH, Tan CK, Wang CY, Liao CH, et al. Catheter-related bacteraemia and infective endocarditis caused by $\mathrm{Ko}$ curia species. Clin Microbiol Infect. 2011; 17(2): 190-2.

Lee JY, Kim SH, Jeong HS, Oh SH, Kim HR, Kim YH, et al. Two cases of peritonitis caused by Kocuria marina in patients undergoing continuous ambulatory peritoneal dialysis. J Clin Microbiol. 2009; 47(10): 3376-8.

Loong SK, Johari J, Seri NAACM, Razak OA, Douadi B, Nasrah SNA, et al. Isolation and identification of an emerging pathogen, Kocuria marina, from Rattus rattus diardii. Trop Biomed. 2016; 33(3): 589-93.

Loong SK, Tan KK, Sulaiman S, Wong PF, AbuBakar S. Draft genome of Bordetella pseudohinzii BH370 isolated from trachea and lung tissues of a laboratory mouse. Genom Data. 2017; 12(2017): 69-70.
Mori N, Nishihara Y, Tayama H, Higuchi A, Aoki Y. Peripherally inserted central catheter-related bloodstream infection caused by Kocuria marina in an elderly man. Infection. 2017; doi:10.1007/ s15010-017-0980-9.

Purty S, Saranathan R, Prashanth K, Narayanan K, Asir J, Devi CS, et al. The expanding spectrum of human infections caused by $\mathrm{Kocu}$ ria species: a case report and literature review. Emerg Microbes Infect. 2013; 2(10): e71.

Tan KK, Tan YC, Chang LY, Lee KW, Nor'e SS, Yee WY, et al. Full genome SNP-based phylogenetic analysis reveals the origin and global spread of Brucella melitensis. BMC Genomics. 2015; 16: 93.

Tan TC, Suresh KG. Predominance of amoeboid forms of Blastocystis hominis in isolates from symptomatic patients. Parasitol Res. 2006; 98(3): 189-93.

Touhami A, Jericho MH, Beveridge TJ. Atomic force microscopy of cell growth and division in Staphylococcus aureus. J Bacteriol. 2004; 186(11): 3286-95. 